\section{Neisseria meningitidis: hit and run}

The pathogen Neisseria meningitidis initially proliferates on the host cell surface in tight aggregates known as microcolonies; infection ensues as individual bacteria detach from the microcolony and disseminate throughout the body. Now, ChamotRooke et al. show that microcolony detachment is regulated by posttranslational modification of type IV pili with phosphoglycerol, which destabilizes the pili bundles that hold the colonies together.

The major component of N. meningitidis type IV pili, PilE, was previously known to be post-translationally modified by phosphoglycerol, but the role of this modification was unknown. Using mass spectrometry and two-dimensional gel electrophoresis, the authors found that a fraction of PilE is modified at Ser93 in bacteria grown on

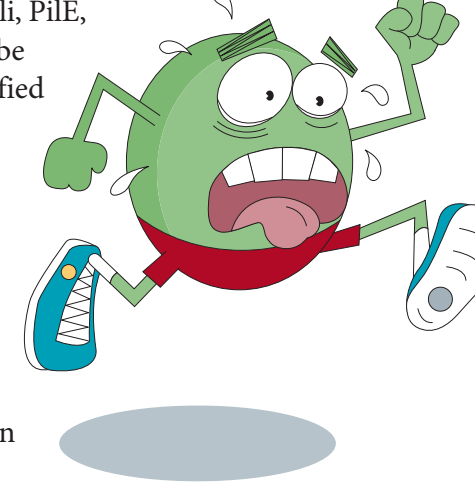

solid medium and that this fraction increases when bacteria are grown in contact with host cells. When the authors deleted the NM_0885 gene, which encodes an orthologue of known phosphoglycerol transferases, they found that the phosphoglycerol modification of PilE was lost. Interestingly, this gene (which the authors renamed $p p t B$, for pilin phosphotransferase B) had been previously shown to be upregulated in response to adhesion to host cells. Furthermore, the authors showed that bacteria lacking $p p t B$ have a reduced ability to detach from an infected monolayer and are deficient in transmigrating across an epithelial monolayer, a step that is known

to be important for invasive

disease. These findings underline the critical role of PptB in cell detachment.

How does modification at one residue lead to such a drastic change in bacterial behaviour? The authors used the known structure of PilE to model the formation of type IV pili bundles and showed that the phosphoglycerol on Ser93 would cause a negative charge to protrude from the pilus, which would destabilize the bundle-forming interactions between pili. This destabilization was confirmed by transmission electron microscopy of pili from wild-type bacteria and $\triangle p p t B$ bacteria. Thus, the increased production of $\mathrm{PptB}$ during the initial stages of microcolony growth leads to an increase in the modification of Ser93 of PilE with phosphoglycerol, and this results in loss of bacterial aggregation. This mechanism may allow $N$. meningitidis to time its spread through the host.

Joanna E. Huddleston

ORIGINAL RESEARCH PAPER Chamot-Rooke, et al. Post-translational modification of pili upon cell contact triggers $N$. meningitidis dissemination. Science 331, 778-782 (2011). 